\title{
Gene screening facilitates diagnosis of complicated symptoms: A case report
}

\author{
HONG DUAN ${ }^{1,2^{*}}$, DI ZHANG ${ }^{1,3^{*}}$, JING CHENG $^{1}, \mathrm{YU} \mathrm{LU}^{1}$ and HUIJUN YUAN ${ }^{1}$ \\ ${ }^{1}$ Department of Surgery, Institute of Otolaryngology, Chinese PLA General Hospital, Beijing 100853; \\ ${ }^{2}$ Department of Otolaryngology, Affiliated Hospital of Inner Mongolia Medical University, Hohhot, \\ Inner Mongolia 010050; ${ }^{3}$ Department of Otorhinolaryngology Head and Neck Surgery, \\ Institute of Otorhinolaryngology, Tianjin First Center Hospital, Tianjin 300192, P.R. China
}

Received April 25, 2016; Accepted May 2, 2017

DOI: $10.3892 / \mathrm{mmr} .2017 .7590$

\begin{abstract}
Gene mutation has an important role in disease pathogenesis; therefore, genetic screening is a useful tool for diagnosis. The present study screened pathogenic genes, ectodysplasin A $(E D A)$ and lamin A/C (LMNA), in a patient with suspected syndromic hearing impairment and various other symptoms including tooth and skin abnormalities. Large-scale sequencing of 438 deafness-associated genes and whole-genome sequencing was also performed. The present findings did not identify copy number variation and mutations in $E D A$; therefore, excluding the possibility of $E D A$-initiated ectodermal dysplasia syndrome. A synonymous mutation in LMNA, possibly due to a splicing abnormality, did not elucidate the pathogenesis of Hutchinson-Gilford progeria syndrome. Whole-genome sequencing revealed copy number variations or mutations in various candidate genes which may elucidate part of the symptoms observed. The copy number variations and mutations were also used to identify single nucleotide variations (SNVs) in crystallin mu $(C R Y M)$, RAB3 GTPase activating protein catalytic subunit $1(R A B 3 G A P l)$ and Wnt family member 10A (WNT10A), implicated in deafness, hypogonadism and tooth/skin abnormalities, respectively. The importance of an existing SNV in $C R Y M$ and a novel SNV in $R A B 3 G A P 1$ in pathogenesis remains to be further elucidated. The WNT10A p.G213S mutation was confirmed to be the
\end{abstract}

Correspondence to: Dr Huijun Yuan, Department of Surgery, Institute of Otolaryngology, Chinese PLA General Hospital, 28 Fuxing Road, Beijing 100853, P.R. China

E-mail: yuanhj301@163.com

*Contributed equally

Abbreviations: HGPS, Hutchinson-Gilford progeria syndrome; NSHL, nonsyndromic hearing loss; SHL, syndromic hearing loss; EDA, ectodermal dysplasia; WGS, whole-genome sequencing; $\mathrm{CNV}$, copy number variation; $\mathrm{SNV}$, single nucleotide variations

Key words: CRYM, LMNA, mutation, RAB3GAPl, sequencing, WNT10A etiological cause of tooth agenesis and ectodermal dysplasia as previously described. It was concluded that a mutation in WNT10A may be the reason for some of the symptoms observed in the patient; however, other genes may also be involved for other symptoms. The findings of the present study provide putative gene mutations that require further investigation in order to determine their roles in pathogenesis.

\section{Introduction}

Among 278 million suffering from deafness worldwide, half are induced by hereditary factors, with 200 to 300 genes having been identified (1). Based on the presence of other symptoms, hearing impairment may be divided into syndromic (SHL, $\sim 30 \%$ ) and nonsyndromic hearing loss (NSHL) $(2,3)$. SHL has $>400$ types of symptoms in the skin, outer ears, eyes and endocrine metabolism. The present study examined a Chinese patient originally suspected to have certain types of SHL. The proband exhibited deafness along with various additional symptoms, including dry loose skin, tooth decay and hypotonia. These phenotypes partially conform to the symptoms of some diseases and syndromes, such as Hutchinson-Gilford progeria syndrome (HGPS) and congenital ectodermal dysplasia syndrome (4-7). HGPS is a rare syndrome characterized by slow growth, prominent eyes, protruding ears, a small chin, hair loss, ageing skin and loss of subcutaneous fat tissue. $(8,9)$ Generally, patients were born normal; however, ageing occurs rapidly, leading to alterations in various organs (10). Some of the proband's symptoms also fall within those of ectodermal dysplasia syndrome (skin and tooth abnormality), whereas other diseases are also possible due to the multiple symptoms of the patient, such as hypogonadism. Genetically, HGPS occurs due to an autosomal dominant inheritance of a mutant lamin A/C (LMNA) gene (11). Ectodermal dysplasia syndrome is attributed to copy number variations (CNVs) or mutations in ectodysplasin A (EDA) gene family members, including $E D A$, EDA receptor $(E D A R)$ and EDAR-associated death domain. $(12,13)$ Therefore, genetic screening is of the utmost importance for diagnosis and treatment.

Given the possibility of HGPS and ectodermal dysplasia syndromes for the patient, sequencing of $L M N A$ exons was performed, followed by $\mathrm{CNV}$ examination of $E D A$ 
gene family members; however, no pathogenic clues were identified. Subsequently, 438 deafness-associated genes were sequenced, in order to identify if these mutations in these genes are associated with the patient's phenotypes. Whole-genome sequencing (WGS) was performed in order to identify potential pathogenic genes that may account for the symptoms observed. The obtained gene list with CNVs or single nucleotide variations (SNVs) was compared with the Human/Mouse Disease Connection database, other SNV-related databases and previous studies in order to identify potential candidate genes. Multiple genes were selected for further analysis.

\section{Case report}

Patient, ethics, consent and permission. Clinical information about the patient, aged 4, male, and the parents was collected in June 2014 , followed by a systemic health check up, under signed informed consent forms to participate and publish the data in accordance with the Ethics Committee of the Chinese PLA General Hospital (Beijing, China). Peripheral blood $(5 \mathrm{ml})$ was collected for genomic DNA (gDNA) isolation.

Sequencing of the HGPS-associated LMNA gene. Primers were based on the NCBI reference sequence of $L M N A$ (Genbank, NM_001282625) and were designed by Shanghai Genesky Biotech Co., Ltd. (Shanghai, China) to amplify exons with $\sim 50$ bp of flanking introns. Primer sequences are presented in Table I. Polymerase chain reaction (PCR) products were purified for sequencing. PCR was performed by Shanghai Genesky Biotech Co., Ltd.

Multiplex ligation-dependent probe amplification (MLPA) for EDA testing. MLPA was used for genetic testing of EDA using P183 kit according to the manufacturer's instructions (MRC-Holland, Amsterdam, Netherlands). DNA was denatured and hybridized with SALSA probe mix, followed by ligation and polymerase chain reaction amplification. Capillary electrophoresis was performed to generate fragment length and peak area using Genemapper software, version 3.0 (Thermo Fisher Scientific, Inc., Waltham, MA, USA). Copy number ratio was denoted as peak area ratio of patient vs. references. A ratio between 0.7 and 1.3 indicated a normal individual, whereas the subject may be diagnosed with ectodermal dysplasia syndrome when the value falls between 1.7 and 2.3. Tests were repeated when the value was near the aforementioned boundaries.

Sequencing of hearing impairment-associated genes. A total of $2 \mu \mathrm{g}$ gDNA was sheared with NEBNext ${ }^{\circledR}$ dsDNA Fragmentase (New England Biolabs, Inc., Ipswich, MA, USA) and end repaired with DNA End Repair Mix (Thermo Fisher Scientific, Inc.), followed by 3'-end adenylation (A-tailing kit; Generay Biotech Co., Ltd., Shanghai, China) and adaptor ligation (NEBNext ${ }^{\circledR}$ Multiplex Oligos for Illumina ${ }^{\circledR}$; New England BioLabs, Inc.) according to the respective manufacturer's instructions. PCR was performed with a primer cocktail using NEBNext ${ }^{\circledR}$ Ultra $^{\mathrm{TM}}$ II DNA Library Prep kit (New England BioLabs, Inc.), followed by purification. The thermocycling conditions were as follows: $98^{\circ} \mathrm{C}$ for $30 \mathrm{sec}, 10$ cycles of $98^{\circ} \mathrm{C}$ for $10 \mathrm{sec}, 60^{\circ} \mathrm{C}$ for $30 \mathrm{sec}$ and $72^{\circ} \mathrm{C}$ for $30 \mathrm{sec}$, followed by $72^{\circ} \mathrm{C}$ for $5 \mathrm{~min}$. Subsequently, the library was pooled, hybridized and purified prior to another round of PCR amplification with the same thermocycling conditions as the ones stated above. Finally, samples were mounted on the Illumina HiSeq 2000 loading unit for sequencing.

WGS procedure. WGS was performed using Illumina TruSeq Nano DNA HT Sample prep kit (Illumina, Inc., San Diego, CA, USA) and HiSeq X system (Illumina, Inc.), according to the manufacturer's protocol. For CNV analysis, sequences of 6 unrelated individuals were used as references. For SNV analysis, the criteria for gene selection were as follows: i) Existing mutations in HGMD; ii) conservation analysis; iii) frequency $<0.001$ in 1,000 genomes or $<0.01$ in its own genome; iv) frequency in ESP6500<0.01; v) single nucleotide polymorphism (SNP) calling quality not L ( L meaning that the SNP cannot be called by either the GAKT or varscan programs), and the ratio of genotyping quality $\mathrm{L}<50 \%$; vi) homology is 1 ; and vii) zero occurrence in the Genesky database. SNP calling was performed using Genome Analysis Toolkit (version 3.7, Broad Institute; software. broadinstitute.org/gatk) and VarScan (version 2.4.0; Genome Institute at Washington University; genome.wustl.edu). An SNP was labeled as ' $\mathrm{H}$ ' if it was identified by both programs, whereas the quality was ' $M$ ' when it was detected by only one. The quality was further downgraded if there were short tandem repeats, indel or homologous sequences flanking the SNP. SNPs labeled as ' $\mathrm{H}$ ' were selected in priority. The overall quality of SNP genotyping was ' $\mathrm{L}$ ' or ' $\mathrm{M}$ '; therefore, if one sample was graded as ' $L$ ' or ' $M$ ' those graded as ' $L$ ' were selected. Genes with sorting intolerant from tolerant (SIFT) values $<0.05$ were selected, as SIFT values indicate the impact of the mutation on protein functions. Additionally, sites with mutation taster scores have a higher probability of mutation. The original gene list was narrowed down according to the aforementioned criteria and genes associated with hearing impairment and HGPS were selected as priority. The list of genes carrying CNV or SNV was matched with the patient's symptoms, including progeria, either in the gene list or the Human-Mouse Disease Connection database (www.informatics.jax.org/mgihome/homepages/human Disease.shtml). Genes of interest were selected for further analysis. An extensive search in existing literature was performed for the refined genes, in order to identify whether the detected mutations in the current patient had been reported to be the pathogenic cause of the phenotypes observed.

Clinical check up of the family. The patient exhibited ageing skin loosening following birth, with limited skin elasticity and subcutaneous fat storage. He exhibited progeroid symptoms without age pigment deposition. Facial skin loosening was moderate; however, there was abnormal development in hair and teeth, with evident tooth decay. The patient had slow reaction to external forces and low muscle tension; however, no abnormality in eyesight, intelligence and overall skeletal development (no bone integration or bone loss) was observed. Additionally, no obvious abnormalities were detected in the nervous system check up. However, the development of the gonads was limited (Fig. 1). 
Table I. Amplification primers of LMNA gene.

\begin{tabular}{cllc}
\hline Exons & \multicolumn{1}{c}{ Forward (5'-3') } & \multicolumn{1}{c}{ Reverse (5'-3') } & Length (bp) \\
\hline 1 & CCAGGAGCAAGCCGAGAG & ACAATTCCCCTTGACACTGC & 990 \\
2 & GGATGCCCTCTCCTGGTAAT & GGCTCTGAAATCAGGTGACAG & 700 \\
3 & CCTGGACCTGTTTCCACAT & TAACCTGGGAGCTGAGTGCT & 680 \\
4 & CCTAGTGGACAGGGAGTTGG & CCTGAGTTGGGCATCACTG & 640 \\
5 & TAGCAGTGATGCCCAACTCA & GCCATCTGACTCCACATCCT & 640 \\
6,7 & CTCTGGGGAAGCTCTGATTG & TCTCACAGCCAAAGAGTCCA & 8,130 \\
8,9 & CAGGGGTGTGTGTAGATGGA & GTTTGCCTACTGGGTGGAGA & 600 \\
10 & AAGTTGCAGGTGGTCACTGG & GAAAGTTCCCACTCCCTTCC & 800 \\
11 & GCACAGAACCACACCTTCCT & GGTGGGCTGTCTAGGACTCA & 520 \\
\hline
\end{tabular}

The patient was not treated after birth. At age 1, the child was diagnosed with severe deafness. No abnormality was observed in skull/temporal bone computed tomography and skull/internal auditory canal magnetic resonance imaging scan prior to artificial cochlea implantation. The patient exhibited high aminotransferase levels; however, no chromosomal abnormality was identified. Following artificial cochlea implantation in 2013, hearing and skin resilience improved. His parents and sister were also examined and were determined to be in healthy condition. The patient was initially suspected to be deaf and have HGPS; therefore, genetic screening was used for diagnosis.

LMNA gene mutation. No mutation was identified in the exons of the HGPS-associated gene LMNA. Exon sequencing of $L M N A$ for the patient and his parents revealed no mutation of $L M N A$ in the family.

MLPA analysis excluded EDA syndrome. EDA peaks for the patient and reference samples are presented in the MLPA histogram of Fig. 2 along with their ratios. Copy number ratios were $\sim 1$, suggesting no change in copy number. Therefore, ectodermal dysplasia syndrome due to dysfunction of EDA was excluded for this patient.

Mutations of deafness-associated genes. Mono-allelic heterozygous mutations were identified in some genes, including coenzyme Q6 monooxygenase, FGFR3, melanogenesis associated transcription factor $(M I T F)$, otoferlin, DNA polymerase $\gamma$ catalytic subunit (POLG) and usherin (USH2A). Mutation of these genes may contribute to other unrelated symptoms. For instance, a POLG mutation may lead to a mitochondrial DNA depletion syndrome, which is characterized by tubulopathy, seizures, respiratory distress, diarrhea and lactic acidosis, which were inconsistent with the majority of the phenotypes observed in the proband. Mutations in some of these genes were also revealed in WGS, and therefore will be discussed further in the following section.

WGS revealed multiple genes that may account for specific symptoms. WGS identified CNV and SNV. From the CNV analysis, 2,653 genes were determined to have half or less
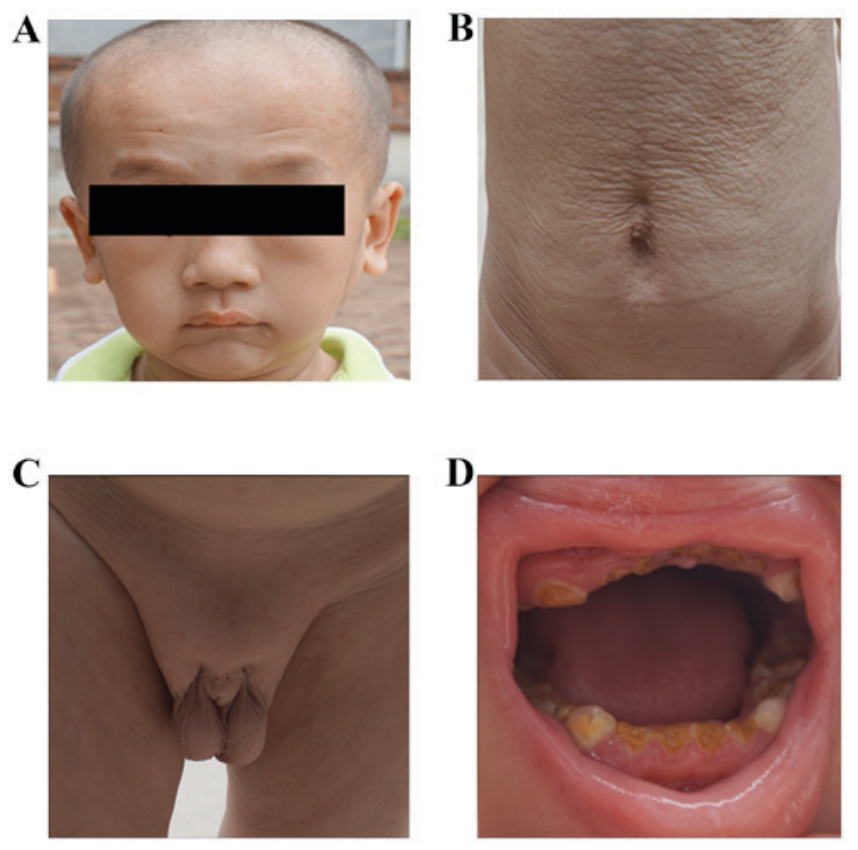

Figure 1. Systemic check up of the patients revealed (A and B) ageing symptoms in the skin, (C) undescended testes and (D) falling off of teeth.

copy numbers. Conversely, the remaining 720 genes had copy numbers $\sim 2$. For the SNV analysis, WGS confirmed the mutations of the aforementioned deafness-associated gene sequencing. Additionally, a full list of genes with autosomal dominant and recessive inheritance patterns were identified.

The genes of interest were identified by searching the lists against the symptoms. Briefly, their mutations were checked in the literature to see what symptoms they may cause. They were categorized according to the phenotypes of the patient in Table II. Some of these genes or their SNVs have been well-characterized and have been identified to be responsible for various diseases. For instance, USH2A and $\mathrm{CDH} 23$ were determined to be involved in the pathogenesis of Usher syndrome $(14,15)$. However, none of the identified genes were capable of simultaneously explaining the majority of the symptoms present in the proband, suggesting that the disease may not be attributed to a monogene and multiple genetic lesions may cooperatively contribute to the presentations in 


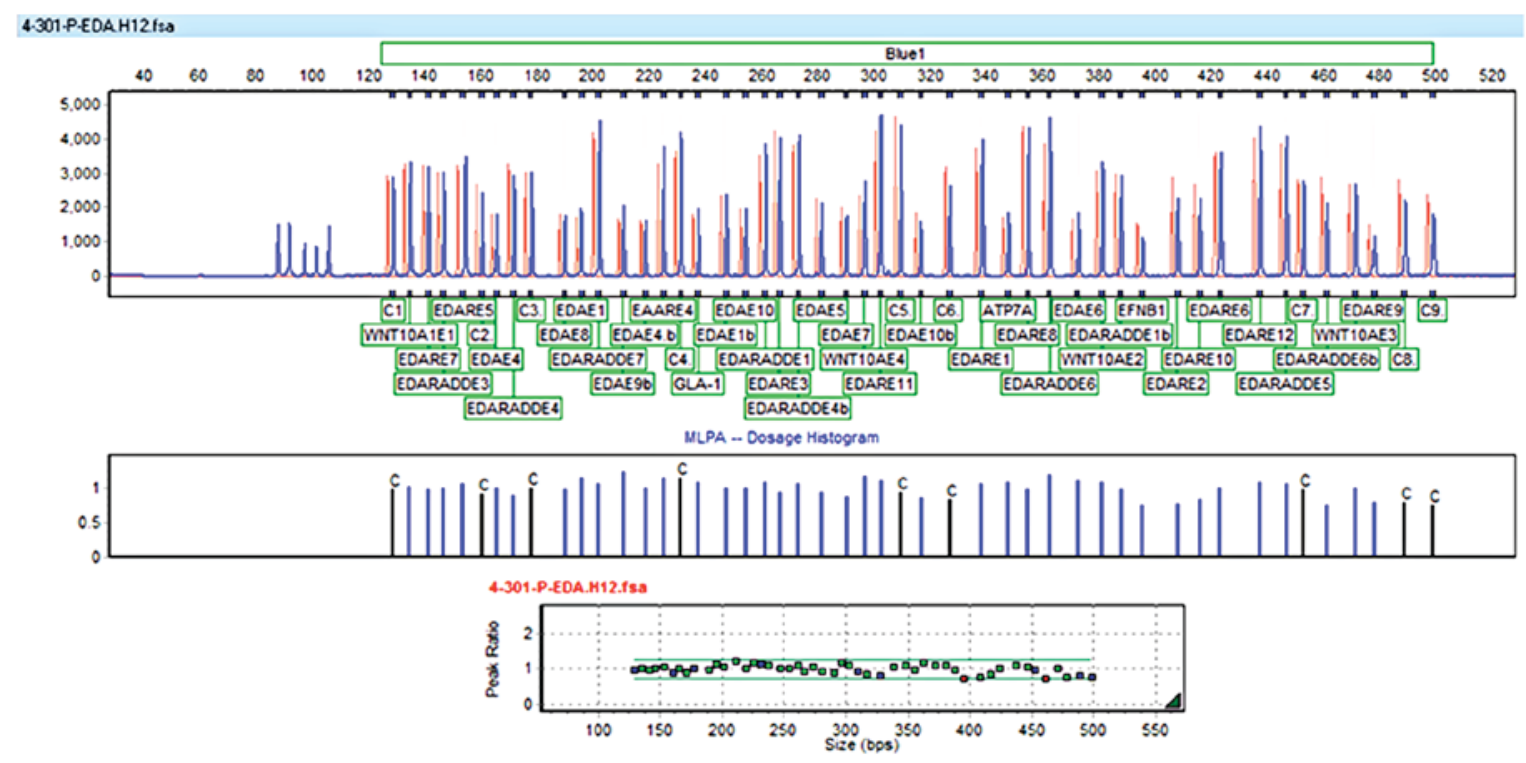

Figure 2. Multiplex ligation-dependent probe amplification analysis of ectodermal dysplasia-associated genes. DNA was denatured and hybridized with SALSA probe mix, followed by ligation and polymerase chain reaction amplification. Capillary electrophoresis was performed to generate fragment length and peak area using GeneMapper version 3.0 software. Copy number ratio is denoted as the ratio of peak area of patient vs. peak area of references. Blue color peaks represent the patient sample, whereas the red color peaks were reference.

the patient. Additionally, the exact SNVs in a number of the listed genes were never reported to be pathogenic in any of the literature, let alone the symptoms presented by the proband in our study. In some cases, the diseases associated with the genes were reported to present major symptoms that were not observed in the present case, such as the Donnai-Barrow syndrome which occurs due to $L R P 2$ mutation (16). Therefore, the majority of the listed genes were filtered out and only SNVs in the $L M N A$, DNA polymerase $\delta 1$, catalytic subunit (POLD1), crystallin mu (CRYM), RAB3 GTPase activating protein catalytic subunit 1 (RAB3GAPI) and Wnt family member 10A (WNT10A) genes were further discussed.

\section{Discussion}

The proband exhibited multiple symptoms, including deafness, ageing and hypogonadism, which partially conform to the symptoms of HGPS and ectodermal dysplasia syndrome. HGPS is a rare hereditary disease which occurs due to autosomal dominant inheritance of mutant $L M N A$, the product of which promotes nuclear membrane deformation and reduces cellular lifespan (17). Previous studies identified the following pathogenic mutations c.1824C $>$ T (p.Gly608Gly), c.1822G $>$ A (p.Gly608Ser), c.1821G >A (p.Val607Val), c.1968+1G >A (18,19). Pathogenic mutations such as c.1824C $>$ T (p.Gly608Gly), do not alter the amino acid sequence; however, they activate a hidden cleavage site, which leads to the deletion of 50 amino acids in the resulting protein $(20,21)$. The present study also identified a synonymous SNV of c.1698C $>$ T, p.His566His (chr1:156107534, NM_001282626, rs4641) at the splice region. However, the allele frequency carrying this SNV is $26.55 \%$ according to ExAC Browser database; therefore, excluding the possibility of rare HGPS in the current patient.

Another gene of interest is $P O L D 1$, the defects of which (serine 605 loss or R507C substitution at exon 13) lead to loss of $\delta$ DNA polymerase activity and impairment of proof-reading exonuclease activity (22-24). This may lead to mandibular dysplasia with deafness and progeroid features (MDP), which is characterized by lipodystrophy, deafness, a small lower jaw, low testosterone levels, claw toes, joint stiffness and hypogonadism (25). However, the nonsynonymous SNV detected in this patient is $1932 \mathrm{C}>\mathrm{G}$ at exon 16 in $P O L D 1$ gene (rs80214209), which has been reported in $>90$ individuals, particularly in East Asia according to ExAC Browser database. Given that the MDP syndrome is an extremely rare syndrome with only 5 reported cases exhibiting a different POLDI SNV (24), it is unlikely that the SNV in POLDI observed in the present study leads to MDP syndrome. The molecular pathogenesis that results in progeria-like features remains to be further elucidated.

Ectodermal dysplasia syndrome occurs partially due to mutations and CNVs in EDAs $(12,13)$. The protein products of these genes participate in signaling pathways that regulate interactions between the ectoderm and mesoderm, critical for the formation of skin, hair, teeth and sweat glands. However, no $\mathrm{CNV}$ or SNV were detected in the EDAs; therefore, this case is unlikely to be $E D A$-caused ectodermal dysplasia. However, it is possible that ectodermal dysplasia may be induced by other gene mutations; for example, c.637G>A, p.G213S of WNT10A.

Deafness may be induced by mutations in multiple genes, such as $U S H 2 A, C D H 23$ as listed in Table II. A dominant splicing alteration (rs189371585) in the CRYM gene was identified as a candidate etiological gene for deafness, with an allele frequency of $0.46 \%$ in the 1,000 genome phase 1 population according to the SNP database (dbSNP). Alterations of CRYM (X315Y and K314T) have been determined to lead to autosomal dominant NSHL (26-28). However, the pathogenic changes previously observed occurred due to an amino acid substitution (26), whereas the present case exhibited alterations in splicing. At present, no existing literature is available 
Table II. Patient phenotypes, associated genes, mutations and diseases attributed to alterations in the genes.

\begin{tabular}{|c|c|c|c|}
\hline \multicolumn{4}{|c|}{ A, Aging/progeria } \\
\hline Gene name & Disease & RefSeq mRNA & $\mathrm{CNV} / \mathrm{SNV}$ \\
\hline LRP1 & NA & NM_002332 & c.12161A>T:p.Y4054F \\
\hline$E D N R A$ & Mandibulofacial dysostosis with alopecia & NM_001166055 & c.503T>C:p.L168P \\
\hline$P O L G$ & Mitochondrial DNA depletion syndrome & NM_001126131 & c.1840T>C:p.Y614H \\
\hline SREBF1 & NA & NM_001005291 & c.547G>A:p.A183T \\
\hline CANX & NA & NM_001024649 & c. $418 \mathrm{C}>$ A:p.L140M \\
\hline BAK1 & NA & NM_001188 & Splicing \\
\hline POLDI & $\begin{array}{l}\text { Mandibular hypoplasia, deafness, progeroid } \\
\text { features and lipodystrophy syndrome }\end{array}$ & NM_001256849 & c.1932C>G:p.D644E \\
\hline$L R P 2$ & Donnai-barrow syndrome & NM_004525 & $\mathrm{CNV}$ ratio $=0.54$ \\
\hline CISD2 & Wolfram syndrome & NM_001008388 & $\mathrm{CNV}$ ratio $=2.03$ \\
\hline VCAM1 & NA & NM_001078 & $\mathrm{CNV}$ ratio $=0.52$ \\
\hline CASP7 & NA & NM_033338 & $\mathrm{CNV}$ ratio $=0.52$ \\
\hline SLC18A2 & NA & NM_003054 & $\mathrm{CNV}$ ratio $=0.60$ \\
\hline IL15 & NA & NM_000585 & $\mathrm{CNV}$ ratio $=0.60$ \\
\hline ADH5 & NA & NM_000671 & $\mathrm{CNV}$ ratio $=0.58$ \\
\hline SLC6A3 & NA & NM_001044 & $\mathrm{CNV}$ ratio $=0.53$ \\
\hline HMGCR & NA & NM_000859 & $\mathrm{CNV}$ ratio $=0.58$ \\
\hline DLD & NA & NM_000108 & $\mathrm{CNV}$ ratio $=0.58$ \\
\hline DDC & NA & NM_001082971 & $\mathrm{CNV}$ ratio $=0.59$ \\
\hline MSRA & NA & NM_001135670 & $\mathrm{CNV}$ ratio $=1.84$ \\
\hline GSN & NA & NM_000177 & $\mathrm{CNV}$ ratio $=0.52$ \\
\hline MLIP & NA & NM_001281746 & $\mathrm{CNV}$ ratio $=0.54$ \\
\hline
\end{tabular}

B, Deafness

\begin{tabular}{|c|c|c|c|}
\hline Gene name & Disease & RefSeq mRNA & $\mathrm{CNV} / \mathrm{SNV}$ \\
\hline USH2A & Usher syndrome II & NM_206933 & $\begin{array}{l}\text { c. } 5608 \mathrm{C}>\mathrm{T}: \mathrm{p} . \mathrm{R} 1870 \mathrm{~W} \\
\text { c.9340C }>\text { T:p.P3114S }\end{array}$ \\
\hline $\mathrm{CDH} 23$ & Usher syndrome & NM_022124 & c.5418C>G:p.D1806E \\
\hline CRYM & & NM_001888.4 & Splicing \\
\hline COQ6 & Nephrotic syndrome & NM_182476 & c.186C>A:p.D62E \\
\hline $\mathrm{FBXO} 2$ & NA & NM_012168 & Splicing \\
\hline STRC & NA & NM_153700 & c.179T>C:p.F60S \\
\hline VPS13B & Cohen syndrome & NM_152564 & c.11884C>G:p.P3962A \\
\hline OTOF & & NM_194248 & c.2123G>A:p.R708Q \\
\hline CISD2 & Wolfram syndrome & & $\mathrm{CNV}$ ratio $=2.03$ \\
\hline$L R P 2$ & Donnai-barrow syndrome & NM_004525 & $\mathrm{CNV}$ ratio $=0.54$ \\
\hline SMCHD1 & Facioscapulohumeal muscular dystrophy & NM_015295 & c.4071T>G:p.I1357M \\
\hline POLDI & $\begin{array}{l}\text { Mandibular hypoplasia, deafness, Progeroid } \\
\text { features, and lipodystrophy syndrome }\end{array}$ & $\begin{array}{l}\text { NM_001256849 } \\
\text { NM_001256849 }\end{array}$ & $\begin{array}{l}\text { c. } 1932 \mathrm{C}>\mathrm{G}: \mathrm{p} . \mathrm{D} 644 \mathrm{E} \\
\text { c.1932C>G:p.D644E }\end{array}$ \\
\hline$P O L G$ & Mitochondrial DNA depletion syndrome & NM_001126131 & c.1840T>C:p.Y614H \\
\hline
\end{tabular}

C, Hypogonadism

\begin{tabular}{llll}
\hline Gene name & \multicolumn{1}{c}{ Disease } & RefSeq mRNA & CNV/SNV \\
\hline RAB3GAP & Warburg syndrome, martsolf syndrome & NM_012233 & c.1175G>A:p.R392Q \\
MITF & Warburg syndrome & NM_198158 & c.1235C>T:p.T412I \\
MAGEL2 & Prader-willi syndrome & NM_019066 & c.1425_1445del:p.475_ \\
& & & 482del
\end{tabular}


Table II. Continued.

C, Hypogonadism

\begin{tabular}{llll}
\hline Gene name & \multicolumn{1}{c}{ Disease } & RefSeq mRNA & CNV/SNV \\
\hline KISS1 & & NM_002256 & c.417delA:p.X139W \\
NRP2 & & NM_201266 & c.1333A $>$ C:p.I445L \\
CISD2 & Wolfram syndrome & NM_001008388 ratio= & CNV \\
& & & c.031485778 \\
POLD1 & Mandibular hypoplasia, deafness, progeroid & NM_001256849 $>$ G:p.D644E \\
Fras1 & Features and lipodystrophy syndrome & NM_025074 & CNV ratio=0.59 \\
& & NM_025074 & c.9356A $>$ G:p.N3119S
\end{tabular}

D, Hypotonia

\begin{tabular}{llll}
\hline Gene name & \multicolumn{1}{c}{ Disease } & RefSeq mRNA & CNV/SNV \\
\hline MAGEL2 & Prader-willi syndrome & NM_019066 & $\begin{array}{l}\text { c.1425_1445del:p.475_ } \\
482 d e l\end{array}$ \\
POLG & Mitochondrial DNA depletion syndrome & NM_001126131 & c.1840T>C:p.Y614H \\
SMCHD1 & Facioscapulohumeral muscular dystrophy & NM_015295 & c.4071T>G:p.I1357M \\
\hline
\end{tabular}

E, Tooth development

\begin{tabular}{llll}
\hline Gene name & \multicolumn{1}{c}{ Disease } & RefSeq mRNA & CNV/SNV \\
\hline EDNRA & Mandibulofacial dysostosis with alopecia & NM_001166055 & c.503T>C:p.L168P \\
POLD1 & $\begin{array}{l}\text { Mandibular hypoplasia, deafness, progeroid } \\
\text { features and lipodystrophy syndrome }\end{array}$ & NM_001256849 & c.1932C>G:p.D644E \\
WNT10A & Odonto-onycho-dermal dysplasia & NM_025216 & c.637G>A:p.G213S \\
\hline
\end{tabular}

Genes in italics were associated with $>1$ symptom. NA, no definite disease is reported to be linked to the gene mutation.

to link this alteration to any pathogenic consequences. Further functional analysis is required to confirm the biological effects of this splicing mutation.

Mutations in several genes may give rise to hypogonadism, including MITF and KiSS-1 metastasis-suppressor. Additionally, diseases associated with mutations in RAB3GAPl include Warburg Micro syndrome and Martsolf syndrome (29-33). Leiden open source variation database archived nonsense mutations at c.1174 that led to the production of truncated protein terminating at p.R392 and contributed to Warburg Micro Syndrome. The novel SNV of c. $1175 \mathrm{G}>\mathrm{A}$ in $R A B 3 G A P 1$ identified in the present study led to a p.R392Q amino acid substitution. Albeit at the same position, no previous studies have reported the pathogenic role of the SNV (p.R392Q) detected in the present study in Warburg Micro syndrome. Additionally, Warburg Micro syndrome is an autosomal recessive disease; however, only the symptom of hypogonadism was consistent with the present case. Therefore, the function of the RAB3GAPI mutation in the current proband remains unclear.

The present study was unable to identify definitive gene lesions that may account for the aforementioned symptoms.
However, an SNV in WNT10A was confirmed to be etiological of tooth agenesis and ectodermal dysplasia. WNT10A produces a protein that triggers the Wnt pathway, which is important for development and oncogenesis. Mutations in the WNT10A gene may lead to aberrant development, such as odonto-onycho-dermal dysplasia, featured by tooth agenesis and ectodermal dysplasia (34-40). Previous studies also demonstrated some overlapping functions of WNT10A with EDAs in inducing hypodontia and ectodermal dysplasia (41-43). Ectodermal dysplasia syndrome exhibits a broad range of symptoms, including but not confined to abnormality of hair growth, absence or malformation of some or all teeth, inability to perspire, impairment or loss of hearing or vision and irregular skin pigmentation. The case presented here conforms to these symptoms in terms of tooth agenesis and hearing loss. Using WGS, a non-synonymous SNV of c.637G >A, p.G213S in WNT10A was detected. This mutation has been previously reported to be the etiological variant leading to tooth agenesis $(35,43)$. In the absence of alterations in EDA members, this variant may also give rise to ectodermal dysplasia (34). Therefore, the gene screening performed in the present study identified the function of the WNT10A mutation 
p.G213S in the induction of tooth agenesis, skin abnormalities and hearing loss.

In conclusion, although further investigation is required to confirm the pathogenic role of some of the SNVs identified in inducing the phenotypes observed, the present study provides an example of the use of genetic screening tools for the diagnosis of a patient with putative syndromic deafness. However, the symptoms were ultimately determined to be attributed to multiple genetic lesions as opposed to a single gene, the present study determined that a WNT10A mutation contributes to the tooth agenesis and ectodermal dysplasia observed in the patient. Additionally, a novel SNV of CRYM and an existing SNV of RAB3GAPl were identified, their function in inducing deafness and hypogonadism require further exploration. The present study provided an example of the use gene screening tools in the diagnosis of a patient with complicated symptoms.

\section{Acknowledgements}

The authors would like to thank the family members for their participation and support in the present study. The present study was supported by the National High Technology Research and Development Program of China (863 Program) (grant no. 2007AA02E466) and the National Natural Science Foundation of China both to Dr. Huijun Yuan (grant no. 30571018).

\section{References}

1. Shearer AE, Eppsteiner RW, Booth KT, Ephraim SS, Gurrola J II, Simpson A, Black-Ziegelbein EA, Joshi S, Ravi H, Giuffre AC, et al: Utilizing ethnic-specific differences in minor allele frequency to recategorize reported pathogenic deafness variants. Am J Hum Genet 95: 445-453, 2014.

2. Yamamoto N, Okuyama H, Hiraumi H, Sakamoto T, Matsuura H and Ito J: The outcome of cochlear implantation for mitochondrial disease patients with syndromic hearing loss. Otol Neurotol 36: e129-e133 2015.

3. Dai ZY, Sun BC, Huang SS, Yuan YY, Zhu YH, Su Y and Dai P. Correlation analysis of phenotype and genotype of GJB2 in patients with non-syndromic hearing loss in China. Gene 570 272-276, 2015.

4. Lamartine J: Towards a new classification of ectodermal dysplasias. Clin Exp Dermatol 28: 351-355, 2003.

5. Yavuz I, Baskan Z, Ulku R, Dulgergil TC, Dari O, Ece A, Yavuz Y and Dari KO: Ectodermal dysplasia: Retrospective study of fifteen cases. Arch Med Res 37: 403-409, 2006.

6. Guler N, Cildir S, Iseri U, Sandalli N and Dilek O: Hypohidrotic ectodermal dysplasia with bilateral impacted teeth at the coronoid process: A case rehabilitated with mini dental implants. Oral Surg Oral Med Oral Pathol Oral Radiol Endod 99: E34-E38, 2005.

7. Yavuz I, Kiralp S and Baskan Z: Hypohidrotic ectodermal dysplasia: A case report. Quintessence Int 39: 81-86, 2008.

8. Fukuo K and Ogihara T: Hutchinson-Gilford progeria syndrome. Ryoikibetsu Shokogun Shirizu: 318-320, 2000 (In Japanese).

9. Sinha JK, Ghosh S and Raghunath M: Progeria: A rare genetic premature ageing disorder. Indian J Med Res 139: 667-674, 2014

10. Mazereeuw-Hautier J, Wilson LC, Mohammed S, Smallwood D, Shackleton S, Atherton DJ and Harper JI: Hutchinson-Gilford progeria syndrome: Clinical findings in three patients carrying the G608G mutation in LMNA and review of the literature. Br J Dermatol 156: 1308-1314, 2007.

11. Vetter U, Pontz B, Zauner E, Brenner RE and Spranger J: Osteogenesis imperfecta: A clinical study of the first ten years of life. Calcif Tissue Int 50: 36-41, 1992.

12. Yasuda M, Kishi C, Yokoyama Y, Amano H and Ishikawa O: Case of X-linked hypohidrotic ectodermal dysplasia with a novel EDA missense mutation. J Dermatol 42: 907-908, 2015.
13. Salas-Alanis JC, Wozniak E, Mein CA, Duran Mckinster CC, Ocampo-Candiani J, Kelsell DP, Hua R, Garza-Rodriguez ML, Choate KA and Barrera Saldaña HA: Mutations in EDA and EDAR genes in a large mexican hispanic cohort with hypohidrotic ectodermal dysplasia. Ann Dermatol 27: 474-477, 2015.

14. Jiang L, Liang X, Li Y, Wang J, Zaneveld JE, Wang H, Xu S, Wang K, Wang B, Chen R and Sui R: Comprehensive molecular diagnosis of 67 Chinese Usher syndrome probands: High rate of ethnicity specific mutations in Chinese USH patients. Orphanet J Rare Dis 10: 110, 2015.

15. Sodi A, Mariottini A, Passerini I, Murro V, Tachyla I, Bianchi B, Menchini U and Torricelli F: MYO7A and USH2A gene sequence variants in Italian patients with Usher syndrome. Mol Vis 20: 1717-1731, 2014.

16. Pober BR, Longoni $M$ and Noonan KM: A review of Donnai-Barrow and facio-oculo-acoustico-renal (DB/FOAR) syndrome: Clinical features and differential diagnosis. Birth Defects Res A Clin Mol Teratol 85: 76-81, 2009.

17. De Sandre-Giovannoli A, Bernard R, Cau P, Navarro C, Amiel J, Boccaccio I, Lyonnet S, Stewart CL, Munnich A, Le Merrer M and Lévy N: Lamin a truncation in hutchinson-gilford progeria. Science 300: 2055, 2003.

18. Kieran MW, Gordon L and Kleinman M: New approaches to progeria. Pediatrics 120: 834-841, 2007.

19. Moulson CL, Fong LG, Gardner JM, Farber EA, Go G, Passariello A, Grange DK, Young SG and Miner JH: Increased progerin expression associated with unusual LMNA mutations causes severe progeroid syndromes. Hum Mutat 28: 882-889, 2007.

20. Chu Y, Xu ZG, Xu Z and Ma L: Hutchinson-Gilford progeria syndrome caused by an LMNA mutation: A case report. Pediatr Dermatol 32: 271-275, 2015.

21. Eriksson M, Brown WT, Gordon LB, Glynn MW, Singer J, Scott L, Erdos MR, Robbins CM, Moses TY and Berglund P: Recurrent de novo point mutations in lamin A cause Hutchinson-Gilford progeria syndrome. Nature 423: 293-298, 2003.

22. Pelosini C, Martinelli S, Ceccarini G, Magno S, Barone I, Basolo A, Fierabracci P, Vitti P, Maffei M, Santini F, et al: Identification of a novel mutation in the polymerase delta 1 (POLD1) gene in a lipodystrophic patient affected by mandibular hypoplasia, deafness, progeroid features (MDPL) syndrome. Metabolism 63: 1385-1389, 2014

23. Weedon MN, Ellard S, Prindle MJ, Caswell R, Lango Allen H, Oram R, Godbole K, Yajnik CS, Sbraccia P, Novelli G, et al: An in-frame deletion at the polymerase active site of POLD1 causes a multisystem disorder with lipodystrophy. Nat Genet 45 : 947-950, 2013.

24. Lessel D, Hisama FM, Szakszon K, Saha B, Sanjuanelo AB, Salbert BA, Steele PD, Baldwin J, Brown WT, Piussan C, et al: POLD1 germline mutations in patients initially diagnosed with werner syndrome. Hum Mutat 36: 1070-1079, 2015.

25. Shastry S, Simha V, Godbole K, Sbraccia P, Melancon S, Yajnik CS, Novelli G, Kroiss M, Garg A, et al: A novel syndrome of mandibular hypoplasia, deafness and progeroid features associated with lipodystrophy, undescended testes, and male hypogonadism. J Clin Endocrinol Metab 95: E192-197, 2010.

26. Abe S, Katagiri T, Saito-Hisaminato A, Usami S, Inoue Y Tsunoda T and Nakamura Y: Identification of CRYM as a candidate responsible for nonsyndromic deafness, through cDNA microarray analysis of human cochlear and vestibular tissues. Am J Hum Genet 72: 73-82, 2003.

27. Yoshimura H, Takumi Y, Nishio SY, Suzuki N, Iwasa Y and Usami S: Deafness gene expression patterns in the mouse cochlea found by microarray analysis. PLoS One 9: e92547, 2014.

28. Oshima A, Suzuki S, Takumi Y, Hashizume K, Abe S and Usami S: CRYM mutations cause deafness through thyroid hormone binding properties in the fibrocytes of the cochlea. J Med Genet 43: e25, 2006.

29. Morris-Rosendahl DJ, Segel R, Born AP, Conrad C, Loeys B, Brooks SS, Müller L, Zeschnigk C, Botti C, Rabinowitz R, et al: New RAB3GAP1 mutations in patients with warburg micro syndrome from different ethnic backgrounds and a possible founder effect in the Danish. Eur J Hum Genet 18: 1100-1106, 2010.

30. Aligianis IA, Morgan NV, Mione M, Johnson CA, Rosser E, Hennekam RC, Adams G, Trembath RC, Pilz DT, Stoodley N, et al: Mutation in Rab3 GTPase-activating protein (RAB3GAP) noncatalytic subunit in a kindred with Martsolf syndrome. Am J Hum Genet 78: 702-707, 2006.

31. Handley MT and Aligianis IA: RAB3GAP1, RAB3GAP2 and RAB18: Disease genes in Micro and Martsolf syndromes. Biochem Soc Trans 40: 1394-1397, 2012. 
32. Handley MT, Morris-Rosendahl DJ, Brown S, Macdonald F, Hardy C, Bem D, Carpanini SM, Borck G, Martorell L, Izzi C, et al: Mutation spectrum in RAB3GAP1, RAB3GAP2 and RAB18 and genotype-phenotype correlations in warburg micro syndrome and Martsolf syndrome. Hum Mutat 34: 686-696, 2013.

33. Asahina M, Endoh Y, Matsubayashi T, Fukuda T and Ogata T: Novel RAB3GAP1 compound heterozygous mutations in Japanese siblings with Warburg Micro syndrome. Brain Dev 38: 337-340, 2016.

34. Clauss F, Waltmann E, Barriere P, Hadj-Rabia S, Manière MC and Schmittbuhl M: Dento-maxillo-facial phenotype and implants-based oral rehabilitation in ectodermal dysplasia with WNT10A gene mutation: Report of a case and literature review. J Craniomaxillofac Surg 42: e346-351, 2014.

35. Mues G, Bonds J, Xiang L, Vieira AR, Seymen F, Klein O and D'Souza RN: The WNT10A gene in ectodermal dysplasias and selective tooth agenesis. Am J Med Genet A 164A: 2455-2460, 2014.

36. Adams BB: Odonto-onycho-dermal dysplasia syndrome. J Am Acad Dermatol 57: 732-733, 2007.

37. Yang J, Wang SK, Choi M, Reid BM, Hu Y, Lee YL, Herzog CR, Kim-Berman H, Lee M, Benke PJ, et al: Taurodontism, variations in tooth number and misshapened crowns in Wnt10a null mice and human kindreds. Mol Genet Genomic Med 3: 40-58, 2015.
38. Kimura R, Watanabe C, Kawaguchi A, Kim YI, Park SB, Maki K, Ishida $\mathrm{H}$ and Yamaguchi T: Common polymorphisms in WNT10A affect tooth morphology as well as hair shape. Hum Mol Genet 24: 2673-2680, 2015.

39. Mostowska A, Biedziak B, Zadurska M, Matuszewska-Trojan S and Jagodziński PP: WNT10A coding variants and maxillary lateral incisor agenesis with associated dental anomalies. Eur J Oral Sci 123: 1-8, 2015

40. Kantaputra P, Kaewgahya M, Jotikasthira D and Kantaputra W: Tricho-odonto-onycho-dermal dysplasia and WNT10A mutations. Am J Med Genet A 164A: 1041-1048, 2014.

41. Bergendal B, Klar J, Stecksén-Blicks C, Norderyd J and Dahl N: Isolated oligodontia associated with mutations in EDARADD, AXIN2, MSX1 and PAX9 genes. Am J Med Genet A 155A: 1616-1622, 2011.

42. Arzoo PS, Klar J, Bergendal B, Norderyd J and Dahl N: WNT10A mutations account for (1/4) of population-based isolated oligodontia and show phenotypic correlations. Am J Med Genet A 164A: 353-359, 2014.

43. He H, Han D, Feng H, Qu H, Song S, Bai B and Zhang Z: Involvement of and interaction between WNT10A and EDA mutations in tooth agenesis cases in the Chinese population. PLoS One 8: e80393, 2013. 